\title{
Light Conscious Sedation in Patients with Previous Acute Myocardial Infarction Needing Exodontia: An Observational Study
}

Fabio Dell'Olio ${ }^{1}$, Saverio Capodiferro ${ }^{1}$, Pantaleo Lorusso ${ }^{2}$, Luisa Limongelli ${ }^{1}$, Angela Tempesta ${ }^{1}$, Maria Massaro $^{2}$, Salvatore Grasso ${ }^{2}$, Gianfranco Favia ${ }^{1}$

1. Interdisciplinary Department of Medicine, University of Bari Aldo Moro, Bari, ITA 2. Emergency Department, University of Bari Aldo Moro, Bari, ITA

Corresponding author: Pantaleo Lorusso, lorussopantaleo@gmail.com

\section{Abstract}

\section{Aim}

This study evaluated a protocol of light conscious sedation for multimodal analgesia in patients with a clinical history of acute myocardial infarction requiring tooth extraction and to assess postoperative pain by using the quantity intake of acetaminophen as the indicator.

\section{Material and methods}

All 50 patients received preliminary anxiolysis via oral chlordemethyldiazepam administration. After 15 to 20 minutes, only patients reporting they were not fully relaxed received additional intravenous diazepam before tooth extraction. Acetaminophen $1000 \mathrm{mg}$ was suggested as the preferred postoperative analgesic drug.

\section{Results}

The studied patients included 39 women and 11 men with a mean age of $69.4 \pm 17.1$ years. They were classified according to the American Society of Anesthesiologists Physical Status classification system as follows: 12 patients belonging to class II, 32 patient to class III, and the remaining six to class IV. Based on the Modified Dental Anxiety Scale, six patients were phobic and seven anxious. Nevertheless, intravenous conscious sedation was needed in 23 patients via diazepam. The first day after surgery, $76 \%$ of patients took acetaminophen, and $58 \%$ took acetaminophen the second day, with a mean two-day total intake of acetaminophen of $1020 \pm 789 \mathrm{mg} /$ day. Stratified statistical analysis was performed and revealed that $60.87 \%$ of patients receiving intravenous diazepam needed to take acetaminophen on the first day after dental extraction in contrast to the $88.89 \%$ of patients who did not receive intravenous diazepam ( $\chi^{2}$ test; $\mathrm{P}=.021$ ).

Received 12/17/2019 Review began 12/19/2019 Review ended 12/27/2019 Published 12/30/2019

๑) Copyright 2019 Dell'Olio et al. This is an open access article distributed under the terms of the Creative Commons Attribution License CC-BY 3.0., which permits unrestricted use, distribution, and reproduction in any medium, provided the original author and source are credited.

\section{Conclusions}

Our data suggest that anxiety related to dental procedures is widespread, although often unmentioned by patients. Moreover, the percentage of patients needing analgesics during the first 24 hours following surgery demonstrated that overall perioperative pain might be controlled by the light conscious sedation protocol for patients with previous acute myocardial infarction proposed in the current study.

Categories: Anesthesiology, Cardiology, Dentistry

Keywords: myocardial infarction, community dentistry, risk assessment, conscious sedation, tooth extraction, oral surgery

\section{Introduction}

Management of anxiety and pain is important for preventing stress-related cardiovascular accidents before, during, and after dental extractions in patients with a clinical history of acute myocardial infarction. Anxiety's manifestations include cardiovascular reactions that may represent a source of stress for patients, such as vasovagal fainting characterized by an increase of heart rate and blood pressure at first with subsequent bradycardia and hypotension-the latter remaining the most common emergency in the dentist's chair [1]. In fact, in such instances, anxiety activates the hypothalamus-pituitary-adrenal axis, inducing cortisol secretion and provides for endogenous catecholamine (mainly epinephrine) release over 10 times more than normal, evoking a strong cardiovascular response with blood pressure and heart rate augmentation, potentially straining a myocardium already weakened by past ischemia [1].

When anxiety is associated with aggression and anger, norepinephrine is released as well [2]. The total amount of circulating catecholamine also includes the exogenous levels injected into tissues during local infiltration of anesthesia. The latter may be involved in causing hemodynamic complications together with 
the endogenous peak; the true role of local anesthetic-related vasoconstrictors is still controversial [3]. Furthermore, some studies reported anxiety-related electrocardiogram (ECG) abnormalities such as ventricular premature contracture and atrial fibrillation that are present in $2.9 \%$ and $1 \%$ of patients, respectively, during local anesthesia execution by a general dentist [2].

Also, pain alerts the hypothalamus-pituitary-adrenal axis, increasing the release of both epinephrine and norepinephrine [2]. This is why inadequate analgesia in patients with previous acute myocardial infarction may represent a risk.

Anxious patients report more pain experienced than non-anxious ones, both during and after a surgical procedure [4]. This can be explained by a strong statistical correlation between the amount of anticipated pain, actual experienced pain, and total intake of anti-inflammatory drugs, with a direct proportion between them. The highest pain is generally reported in the first 24 hours after surgery [5]. There is also strong evidence for benzodiazepines (BDZ) as reducers of preoperative anxiety, experienced pain, and postoperative pain [5-6].

The current study aims to present a light conscious sedation protocol able to provide multimodal analgesia in patients with a history of acute myocardial infarction requiring tooth extraction and to assess postoperative pain using the quantity of acetaminophen intake as an indicator.

\section{Materials And Methods}

The authors studied 50 patients referred to the Complex Operating Unit of Odontostomatology, Aldo Moro University of Bari, needing teeth extracted from 2017 to 2018 and matching the following inclusion criteria: a clinical history of acute myocardial infarction occurring at least one year prior; ongoing antiplatelet therapy without any possible suspension for dental procedure; acetaminophen as a possible drug administrable as a postoperative analgesic; no allergic reactions to lidocaine, mepivacaine, diazepam, and acetaminophen; and negative anamnesis for neuropsychiatric disease. All patients provided informed consent to join the study.

A panoramic radiogram, standard blood tests, ECG, and an anesthesiological and cardiologic evaluation were performed for all patients. Each patient was classified according to the American Society of Anesthesiologists Physical Status (ASA PS) classification system, Modified Dental Anxiety Scale (MDAS), and Newman's test [7-8]. For easier management of intra- and postoperative bleeding, extraction of only up to two teeth was suggested by a cardiologist. To stabilize cardiovascular parameters, chlordemethyldiazepam (CDDZ, En) drops in amounts varying from $0.5 \mathrm{mg}$ to $1 \mathrm{mg}$ were administered orally to patients with an MDAS score lower than 14, while anxious (MDAS $\geqslant 14$ ) and phobic (MDAS $\geqslant 18$ ) patients received $1 \mathrm{mg}$ to 2 mg of CDDZ. Patient vital parameters were continuously monitored by a pulse oximeter, noninvasive blood pressure measurement every five minutes, and also by a three-lead continuous ECG system for the entire procedure. Patients reporting to be not fully relaxed after a waiting period of 15 to 20 minutes after CDDZ administration received additional intravenous diazepam using the following administration algorithm: an initial infusion of $2 \mathrm{mg}$ followed by $1 \mathrm{mg}$ every two minutes until reaching maximum individual tranquility (the end-point of sedation) or a maximum limit of $8 \mathrm{mg}$. Assessment of sedation was made one minute after each diazepam administration, by using an analogic scale ranging from 0 (absence of tranquility) to 10 (maximum individual tranquility) [9]. After achieving the end-point of sedation, tooth extractions were performed after topical anesthesia using a lidocaine $10 \mathrm{~g} / 100 \mathrm{~mL}$ spray and injection of mepivacaine $3 \%$ (1.8 $\mathrm{mL}$ vials) without adrenaline. Patients underwent a postoperative Newman's test to assess attention, motility, and cognitive level and were discharged one hour after the test was negative (omission of $\geqslant 7$ points). Acetaminophen $1000 \mathrm{mg}$ tablets (maximum of three/day) was suggested as postoperative analgesic therapy. We asked patients to record the total number of acetaminophen tablets taken in the first two days after surgery. This total was reported during a phone interview that would be performed during the third day or the one-week follow-up. The current study was designed to measure the actual pain experienced by patients via the consumption of acetaminophen to bypass visual analogical scale-related biases.

All data regarding age, sex, ASA PS classification, MDAS score, doses of CDDZ and diazepam, and acetaminophen tablets consumption during the first and the second day after surgery were collected for statistical analysis with a type I error estimated at $5 \%(\mathrm{P}<.05)$.

The study was carried out in accordance with the principles of the Declaration of Helsinki. Patients provided informed consent for diagnostic and therapeutic procedures performed for research purposes.

\section{Results}

Table 1 summarizes all the results obtained in the current study. The patients were 39 women and 11 men with a mean age of 69.4 years and a standard deviation (SD) of 17.1 years. The ASA PS classification of risk assessed 12 patients as ASA II, 32 as ASA III, and six as ASA IV. According to the MDAS score evaluation, six patients were phobic and seven anxious, while 37 had an MDAS score lower than 14. CDDZ was administered to all patients $(0.8 \mathrm{mg} \pm 0.46 \mathrm{mg}$ ) while, despite MDAS scores, intravenous conscious sedation was needed in 23 patients instead of the 13 expected from the diazepam group (DG); the remaining individuals requiring 


\section{Cureus}

this were from the no-diazepam-group (NDG). During the first day after surgery, $76 \%$ of patients took acetaminophen while $58 \%$ did during the second, giving a mean two-day total intake of acetaminophen of $1020 \pm 789 \mathrm{mg}$ /day. Stratifying patients between DG and NDG, we observed that DG received on average $1.022 \mathrm{mg}$ of CDDZ (SD = $0.574 \mathrm{mg}$ ) while NDG received $0.611 \mathrm{mg}(\mathrm{SD}=0.212 \mathrm{mg})$; no statistical difference was found between the two groups (t-test; $P=.999$ ). $D G$ received an average quantity of $2.444 \mathrm{mg}$ of diazepam $(\mathrm{SD}=1.303 \mathrm{mg}$ ). We found that $88.89 \%$ of NDG needed acetaminophen in the first day after dental extraction, in contrast to $60.87 \%$ of DG (summarized in Table 2); this was a statistically significant difference $(\chi 2$ test; $P=.021)$. During the second day, the proportion of acetaminophen use was statistically similar in both groups $(\chi 2$ test; $P=.704)$. The mean two-day total intake of acetaminophen between groups was statistically similar (t-test; $\mathrm{P}=.471$ ): $\mathrm{DG}$ took $978 \mathrm{mg} /$ day $(\mathrm{SD}=859 \mathrm{mg} /$ day) while NDG took 1056 $\mathrm{mg} /$ day (SD $=738 \mathrm{mg} /$ day). Hemorrhagic complications never occurred.

\begin{tabular}{|l|l|}
\hline Characteristic & Number \\
\hline Sex (M/F) & $11 / 39$ \\
\hline Age $($ mean \pm SD) & $69.4 \pm 17.1$ years \\
ASA PS classification (II/III/IV) & $12 / 32 / 4$ \\
\hline MDAS score (<14/14-17/ $\geq 18)$ & $37 / 7 / 6$ \\
\hline Diazepam/No Diazepam Group & $23 / 27$ \\
\hline Patients requiring analgesic (Day 1$)$ & $38(76 \%)$ \\
\hline Patients requiring analgesic (Day 2) & $29(58 \%)$ \\
\hline Mean two-day acetaminophen intake (mean \pm SD) & $1020 \pm 789 \mathrm{mg} / \mathrm{day}$ \\
\hline
\end{tabular}

\section{TABLE 1: Sample characteristics}

Sample characteristics: 37 patients had a low MDAS score but 14 of them required supplementary anxiolysis before surgery regardless.

Abbreviations: ASA PS, American Society of Anesthesiologists Physical Status; F, female; M, male; MDAS, modified dental anxiety scale; SD, standard deviation.

\begin{tabular}{|c|c|c|c|}
\hline & No Diazepam Group & Diazepam Group & P-value \\
\hline Day 1 & $88.89 \%$ & $60.87 \%$ & .021 \\
\hline Day 2 & $60.87 \%$ & $55.56 \%$ & .704 \\
\hline Mean & $1056 \pm 738 \mathrm{mg} /$ day & $978 \pm 859 \mathrm{mg} / \mathrm{day}$ & .471 \\
\hline
\end{tabular}

\section{TABLE 2: Acetaminophen intake between no-diazepam and diazepam group}

The proportion of patients that needed acetaminophen is reported for the first (Day 1) and the second (Day 2) day after surgery. The bottom line lists the daily mean intake of acetaminophen (mean \pm SD) for an average patient in each group.

Abbreviation: SD, standard deviation.

\section{Discussion}

This is a single-center prospective study performed in accordance with the definition of conscious sedation given by the General Dental Council, which is "keep patient conscious, with his protective reflexes and able to respond to verbal commands” [10]. Furthermore, the administration of a BDZ with primary anxiolytic activity is advisable before dental procedures. Usually, diazepam and CDDZ are preferable to midazolam, as in the present study, as diazepam acts on the $\alpha 2$ subunit of the GABAA receptor, providing a strong anxiolytic effect and weak sedative effects, while midazolam targets the $\alpha 1$ subunit, thereby being strongly sedative and weakly anxiolytic [9,11]. Eventually, the $\alpha 2$ subunit is also involved in the prevention of hyperalgesia, providing a low analgesic effect, prolonged by the long half-life of diazepam, ranging between 30 and 56 hours [12]. This study shows that the percentage of patients of DG suffering very little in the first 24 hours from surgery and needing no rescue analgesic (acetaminophen) was about 20\% more than those in the NDG group. This difference disappeared during the second day, mainly because some of NDG patients 
refused to take acetaminophen and instead waited for spontaneous reduction of pain. These different behaviors during the second day after surgery explain why the total consumption of acetaminophen was statistically similar between the two groups.

The combination of conscious sedation with the endpoint of maximum individual tranquility and correct local anesthesia provides a perioperative experience that allows safe treatment of at-risk patients. This study differs from other reports as it enrolled mainly patients with an ASA score $\geqslant$ III (76\%). These patients have been treated in a fashion providing a continuous stabilization of vital parameters during the entire perioperative period.

\section{Conclusions}

Our data suggest that anxiety related to dental procedures is widespread, though often unreported by patients. Perioperative pain may be controlled by the suggested light conscious sedation protocol in patients including those with previous acute myocardial infarction. Further studies could confirm the encouraging results obtained in this study possibly in randomized controlled trials to provide more evidence and better treatment of patients with myocardial ischemia.

\section{Additional Information}

\section{Disclosures}

Human subjects: Consent was obtained by all participants in this study. Animal subjects: All authors have confirmed that this study did not involve animal subjects or tissue. Conflicts of interest: In compliance with the ICMJE uniform disclosure form, all authors declare the following: Payment/services info: All authors have declared that no financial support was received from any organization for the submitted work. Financial relationships: All authors have declared that they have no financial relationships at present or within the previous three years with any organizations that might have an interest in the submitted work. Other relationships: All authors have declared that there are no other relationships or activities that could appear to have influenced the submitted work.

\section{References}

1. Malamed S: Pain and anxiety in dentistry. Sedation: A Guide to Patient Management. Young ER (ed): Mosby, St. Louis; 2003. 10:2344/0003. 10.2344/0003-3006(2005)52[43:sagtpm]2.0.CO;2

2. Liau FL, Kok S-H, Lee J-J, et al.: Cardiovascular influence of dental anxiety during local anesthesia for tooth extraction. Oral Surg Oral Med Oral Pathol Oral Radiol. 2008, 105:16-26. 10.1016/j.tripleo.2007.03.015

3. Meyer F-U: Haemodynamic changes under emotional stress following a minor surgical procedure under local anaesthesia. Int J Oral Maxillofac Surg. 1987, 16:688-694. 10.1016/S0901-5027(87)80054-1

4. Eli I, Schwartz-Arad D, Baht R, Ben-Tuvim H: Effect of anxiety on the experience of pain in implant insertion. Clin Oral Implants Res. 2003, 14:115-118. 10.1034/j.1600-0501.2003.140115.x

5. Beaudette JR, Fritz PC, Sullivan PJ, Piccini A, Ward WE: Investigation of factors that influence pain experienced and the use of pain medication following periodontal surgery. J Clin Periodontol. 2018, 45:578585. 10.1111/jcpe.12885

6. Wilson TD, McNeil DW, Kyle BN, Weaver BD, Graves RW: Effects of conscious sedation on patient recall of anxiety and pain after oral surgery. Oral Surg Oral Med Oral Pathol Oral Radiol. 2014, 117:277-282. 10.1016/j.0000.2013.11.489

7. Facco E, Gumirato E, Humphris G, et al.: Modified Dental Anxiety Scale: validation of the Italian version . Minerva Stomatol. 2015, 64:295-307.

8. Newman MG, Trieger N, Miller JC: Measuring recovery from anesthesia - a simple test . Anesth Analg. 1969, 48:136-40. 10.1213/00000539-196901000-00028

9. Zanette G, Manani G, Favero L, et al.: Conscious sedation with diazepam and midazolam for dental patient: priority to diazepam. Minerva Stomatol. 2013, 62:355-374.

10. Zanette G, Robb N, Facco E, Zanette L, Manani G: Sedation in dentistry: current sedation practice in Italy . Eur J Anaesthesiol. 2007, 24:198-200. 10.1017/S0265021506001785

11. Vinkers $\mathrm{CH}$, Klanker M, Groenink L, et al.: Dissociating anxiolytic and sedative effects of GABAAergic drugs using temperature and locomotor responses to acute stress. Psychopharmacology (Berl). 2009, 204:299-311. 10.1007/s00213-009-1460-4

12. Rudolph U, Knoflach F: Beyond classical benzodiazepines: novel therapeutic potential of GABAA receptor subtypes. Nat Rev Drug Discov. 2011, 10:685-697. 10.1038/nrd3502 